\title{
Sistematización de la Atención de Enfermería: Realidad o utopía desde la perspectiva de académicos de la Escuela de Enfermería "Magalhães Barata"
} Systematization of Nursing Assistance: Reality or utopia from the perspective of academics "Magalhães Barata” Nursing School

\section{Sistematização da Assistência de Enfermagem: Realidade ou Utopia sob a ótica de acadêmicos da Escola de Enfermagem "Magalhães Barata"}

\author{
Neiva José da Luz Dias Júnior ${ }^{1}$, Lourdes Oliveira Gomes ${ }^{2}$, Mary Elizabeth de Santana ${ }^{3}$, \\ Sandra Helena Isse Polaro ${ }^{4}$
}

${ }^{1}$ Enfermeiro. Mestrando do Programa de Pós-Graduação Mestrado em Enfermagem da Universidade Federal do Pará (UFPA). Belém, Pará, Brasil. E-mail: neiva_jr@hotmail.com

${ }^{2}$ Enfermeira. Mestre em Biologia de Agentes Infecciosos e Parasitários pela Universidade Federal do Pará (UFPA). Belém, Pará, Brasil. ${ }^{3}$ Enfermeira. Doutora em Enfermagem Fundamental pela Escola de Enfermagem de Ribeirão Preto (EERP) da Universidade de São Paulo (USP). Professora Titular da Escola de Enfermagem Magalhães Barata (EEMB) da Universidade do Estado do Pará (UEPA) e Professora Adjunto III da Faculdade de Enfermagem da UFPA. Belém, Pará, Brasil.

${ }^{4}$ Enfermeira. Doutora em Enfermagem pela Universidade Federal de Santa Catarina (UFSC). Professora Adjunto III da Faculdade de Enfermagem da UFPA. Belém, Pará, Brasil.

Cómo citar este artículo en edición digital: Dias Junior, N.J.L., Gomes, L.O., Santana, M.E., \& Polaro, S.H.I. (2017). Sistematización de la Atención de Enfermería: Realidad o utopía desde la perspectiva de académicos de la Escuela de Enfermería "Magalhães Barata”. Cultura de los Cuidados (Edición digital), 21(48). Recuperado de < http://dx.doi.org/10.14198/cuid.2017.48.16>

\section{Correspondência:}

Neiva José da Luz Dias Júnior. Rua Santa Cruz nº 04, Telegrafo. Belém/PA/Brasil. CEP: 66115-080.

Correo electrónico: neiva_jr@hotmail.com

Recibido: 22/12/2016; Aceptado: 10/03/2017

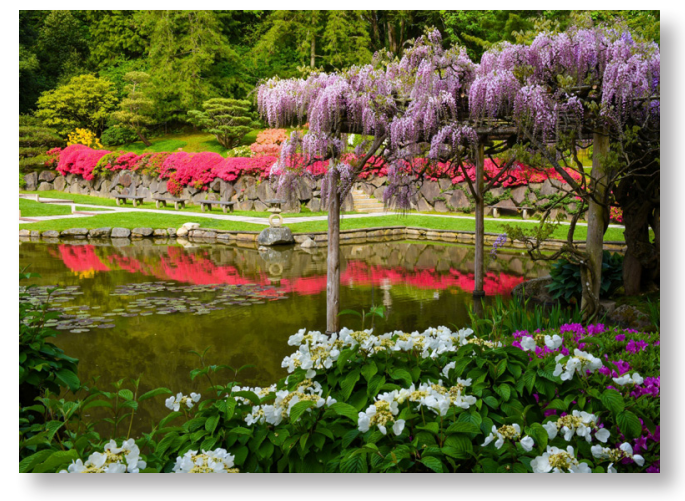

\section{ABSTRACT}

This study focus on a descriptive work by a qualitative approach in order to analyze the perception of the academics in Magalhães Barata Nursing School (MBNS) over systematization of nursing assistance (SNA). This research took place at Magalhães Barata Nursing School of Pará State University. Twenty nur- sing graduates were subject of this research. Collection of data was made by an interview composed of five open questions. There are four categories which emerged through the process: Defining SNA; The SNA teaching in MBNS and its trainee area difficulties; The importance of SNA to professional development and SNA:Reality or utopia?. It becomes evident the need for association between the academy and the health service so that the difficulties will be overcome in both.

Keywords: Nursing,nursing education research, nursing theory, students, nursing.

\section{RESUMEN}

Este estudio tiene como objetivos: analizar las percepciones de los académicos de la Escuela de Enfermería "Magalhães Barata" (EEMB) 
acerca de la Sistematización de la Atención de Enfermería (SAE). Se realizó un estudio descriptivo con abordaje cualitativo. El escenario fue la EEMB de la Universidad del Estado de Pará, 20 sujetos participaron. La técnica de recolección de datos se aplicó un cuestionario con cinco preguntas abiertas. Después de examinar surgido cuatro categorías: Definición de SAE; SAE Enseñanza en EEMB y sus dificultades en los ámbitos de la formación; la importancia del desarrollo profesional de la SAE y SAE: Realidad o utopía?. Se hace evidente la necesidad de asociación entre la academia y el servicio de salud a fin de que las dificultades se superarán en ambos.

Palabras Clave: Enfermería, investigación en Enfermería, teoría de Enfermería, estudiantes de Enfermería.

\section{RESUMO}

Este estudo tem como objetivo: analisar a percepção dos acadêmicos da Escola de Enfermagem "Magalhães Barata” (EEMB) acerca da sistematização da assistência de enfermagem (SAE). Realizou-se um estudo descritivo, com abordagem qualitativa. O cenário foi a EEMB da Universidade do Estado do Pará, participaram 20 sujeitos. A técnica de coleta de dados foi aplicação de formulário com cinco perguntas abertas. Após análise emergiram quatro categorias: Definindo SAE; O ensino da SAE na EEMB e suas dificuldades nos campos de estágio; A importância da SAE para o desenvolvimento profissional e SAE: Realidade ou Utopia?. Torna-se evidente a necessidade da associação entre a academia e o serviço de saúde de modo que as dificuldades encontradas em ambos sejam superadas.

Palavras-Chave: Enfermagem; Pesquisa em educação de enfermagem, teoria de enfermagem, estudantes de enfermagem.

\section{INTRODUÇÃO}

A evolução da enfermagem e sua consolidação enquanto ciência é caracterizada pela construção de um corpo de conhecimento próprio no decorrer de sua história. Nos anos 70 houve uma preocupação das enfermeiras com o desenvolvimento de teorias de enfermagem, como um meio de estabelecer a enfermagem como profissão (Ângelo, Forcella, e Fukuda, 1995).

As teorias constituem um modo sistemático de olhar o mundo para descrevê-lo, explicá-lo, prevê-lo ou controlá-lo. É dessa forma que a teoria de enfermagem é definida como uma conceitualização articulada e comunicada da realidade, inventada ou descoberta, com a finalidade de descrever, explicar, prever ou prescrever o cuidado de enfermagem (Fialho, Pagliuca, e Soares, 2002).

Dentro desse corpo de conhecimentos, baseados em teorias de enfermagem que surge a Sistematização da Assistência de Enfermagem - SAE, sendo uma representação do mecanismo por meio do qual esses conhecimentos são aplicados na prática profissional do enfermeiro.

A SAE é uma forma sistemática e dinâmica de prestar os cuidados de enfermagem. Esse processo consiste em cinco etapas inter-relacionadas: investigação, diagnóstico, planejamento, implementação e avaliação (Alfaro-Lefevre, 2014).

Para Horta (1979), a SAE inicia-se com a elaboração do Histórico e/ou Investigação de Enfermagem, que consiste em roteiro sistematizado para o levantamento de dados tornando possível a identificação de problemas. Posteriormente institui - se a fase de identificação do Diagnóstico de Enfermagem, que consiste no julgamento clínico das respostas do indivíduo, da família e da comunidade quanto aos processos vitais, aos problemas de saúde atuais 
ou de risco (NANDA, 2015). O planejamento de enfermagem, considerado como a terceira etapa da SAE, é um roteiro diário que coordena as ações da equipe de enfermagem de cuidado e atendimento das necessidades básicas e especificas do ser humano. A quarta etapa do processo é a implementação do cuidado que significa colocar o plano em ação, sempre refletindo sobre o que está fazendo, investigando a situação atual da pessoa antes de intervir; realizar as intervenções e reinvestigar para determinar as respostas iniciais; deve-se além de registrar, comunicar o plano. Por fim, chega-se ao processo de avaliação dos resultados esperados, onde é realizada a evolução do paciente, ou seja, o relato diário das alterações ocorridas ao cliente durante o tempo que estiver sob assistência profissional (Horta,1979).

Durante a Graduação em Enfermagem se desperta o interesse em compreender o que diferencia a profissão das demais profissões direcionadas à saúde, no que tange à maneira de exercê-la, sobretudo no que diz respeito às atribuições da equipe de enfermagem.

Após o primeiro contato com a SAE, é possível compreender que o enfermeiro possui formação e competência para aplicar seus conhecimentos técnico-científicos na prática assistencial, de maneira a realizar seu trabalho de forma coerente e coesa.

A SAE torna-se atividade privativa do enfermeiro no planejamento das ações de cuidado, atribuição essa que o diferencia dos outros trabalhadores da equipe. Assim, a presente pesquisa teve por objetivo identificar a visão dos futuros profissionais de enfermagem acerca do exercício profissional centrado na execução da SAE durante a assistência ao paciente.

\section{METODOLOGIA}

Trata-se de um estudo exploratório e descritivo com abordagem qualitativa, tendo

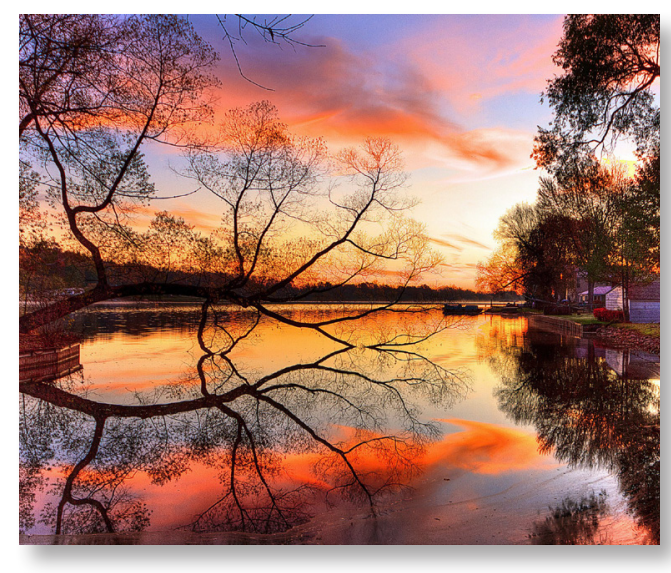

como local de estudo a Escola de Enfermagem Magalhães Barata (EEMB) da Universidade do Estado do Pará (UEPA), localizada na cidade de Belém, no estado do Pará, Brasil.

Considerou-se como critério de inclusão ser acadêmico de Graduação em Enfermagem e estar cursando o segundo bloco de disciplinas do $4^{\circ}$ e $5^{\circ}$ ano do curso, totalizando uma amostra de 70 possíveis participantes. Após a transcrição da décima sexta entrevista foi possível perceber a saturação dos dados, que foi confirmada com a realização de mais quatro entrevistas, totalizando uma amostra final de 20 participantes.

A coleta de informações foi realizada através de entrevista semiestruturada composta por perguntas abertas. Os entrevistados foram identificados por códigos alfa numéricos com a ordem de participação na pesquisa, preservando o anonimato dos mesmos. Todas as entrevistas foram gravadas e posteriormente, transcritas na íntegra. Todos os participantes tiveram seus direitos preservados através de assinatura do Termo de Consentimento Livre e Esclarecido e do Termo de Consentimento para Gravações.

As informações coletadas foram analisadas seguindo as etapas do método de Análise Temática de Conteúdo, que consiste em: pré-análise: consistiu na organização do material, 
com objetivo de sistematizar as ideias iniciais; exploração do material: momento em que foram definidas as categorias; tratamento dos resultados obtidos e interpretação: nessa etapa foi realizada a interpretação das informações com intenção de colocar em relevo as informações obtidas e contextualizá-las com a literatura (Minayo, 2014).

Os aspectos éticos e legais foram respeitados e assegurados pela Resolução 196/96 do Conselho Nacional de Ética em Pesquisa Brasileiro, após aprovação e parecer consubstanciado do Comitê de Ética em Pesquisa com Seres Humanos do Curso de Graduação em Enfermagem da Universidade do Estado do Pará, sob o número nº 0036.0.321.000-10.

\section{RESULTADOS E DISCUSSÃO}

Foram entrevistados vinte acadêmicos de enfermagem, sendo nove e onze acadêmicos do $5^{\circ}$ e $4^{\circ}$ ano respectivamente. Sendo dezesseis do sexo feminino e quatro do sexo masculino, tendo uma média de idade entre 20 e 22 anos. A partir das leituras e reflexões do material produzido através das entrevistas emergiram três categorias temáticas, sendo elas: $\mathrm{O}$ ensino da SAE na EEMB/UEPA e suas dificuldades nos campos de estágio; A importância da SAE para o desenvolvimento profissional e SAE: Realidade ou Utopia na prática de enfermagem?

\section{O Ensino da SAE e suas dificuldades nos campos de estágio.}

Para os acadêmicos de enfermagem utilizar a SAE auxilia no aprendizado do profissionalismo em sua prática. Os acadêmicos foram unânimes ao afirmarem que poucas vezes realizaram a SAE de forma completa no campo de prática, sendo na maioria das vezes vivenciada de forma parcial ou fragmentada.
[...] nos campos de estágio a SAE dificilmente é executada e quando executada não há o desenvolvimento integral das etapas sendo muitas vezes só realizada a anamnese, diagnóstico e prescrição sendo inexistente principalmente a avaliação [...]. Além disso, podemos perceber que a maioria dos profissionais não apresenta domínio ou não tem tempo para desenvolver a SAE nas instituições. (ACD 1)

Sabe-se da importância da aplicação do processo de enfermagem, mas é notória a resistência por parte dos enfermeiros em aceitar e aplicar esse método científico de trabalho. Os motivos são inúmeros e superficiais, tais como a falta de tempo, o quantitativo reduzido de profissionais na equipe de enfermagem e a abordagem teórica sem aplicabilidade prática (Waldow, 1988).

As principais dificuldades para aplicar a SAE nos campos de estágio são a não adesão dos campos de estágio à SAE e dos nossos docentes que não se preocupam em desenvolver a SAE no dia a dia das práticas. (ACD 8 )

De modo geral, a SAE é ensinada com padrões que remetem e denotam resquícios de uma visão tecnicista. São transmitidos conteúdos acerca das etapas da SAE e os procedimentos envolvidos no processo, e a prática da formação é desenvolvida em momentos e locais diferentes.

Quanto ao ensino da SAE na EEMB da Universidade do Estado do Pará, os acadêmicos denotam uma visão crítica em relação ao contexto da formação, demonstram uma carência em relação ao ensino nesse contexto:

Na teoria a SAE é muito bem explicada e enfatizada por alguns docentes, mas a prática torna-se muito mais difí- 
cil, pois nos campos de prática ela praticamente não existe, fazendo com que os docentes deixem a SAE em segundo plano no campo prático. (ACD 7).

A prática é a base para o desenvolvimento da teoria de enfermagem, e essa teoria deve ser validada na prática. A teoria tem suas raízes na prática, sendo refinada pela pesquisa e reaplicada na prática (Ojeda, Santos e Eidt, 2004; Mcewen e Wills, 2015).

\section{A Importância da SAE para o desenvolvi- mento profissional.}

A importância da prática da SAE na vida acadêmica dos entrevistados apresenta-se como reflexo no seu futuro posicionamento quanto profissional sobre o desenvolvimento da SAE.

A SAE é fundamental para prestar uma assistência humanizada, com eficiência e eficácia, pois é a mola mestra para fortalecer a enfermagem como ciência e arte do cuidar e sua aplicação garante o reconhecimento dos profissionais que utilizam como ferramenta de trabalho (ACD 3).

Como a própria definição já diz é da SAE que emerge a assistência de enfermagem, bem como da qualidade que esta vai ser prestada, visando objetivos/ Metas especificas para o bem-estar de cada paciente. (ACD 12)

$O$ cuidado prestado de forma sistematizada, organizada e registrada reflete a qualidade da assistência que se apresenta com coerência beneficiando o tratamento do cliente. Além disso, a linguagem padronizada, e compartilhada, utilizada no desenvolvimento da SAE promove autonomia no planejamento do cuidado e diferencia a atuação da enfermagem (Cunha e Barros, 2005; Garcia e Nobrega, 2009).
É vital para desenvolver e crescer a profissão. Aplicar a SAE é melhorar a assistência e o ensino (ACD 6).

A SAE é importante para a profissão, uma vez que legitima esta como ciência e oportuniza o cuidar do paciente em toda sua integralidade (ACD 10).

A cientificidade que a SAE traz para a profissão de enfermagem é visivelmente notada pelos acadêmicos e concerne com o entendimento de que a sistematização da assistência não só permite como também fortalece a enfermagem como ciência, proporcionando uma atividade profissional mais independente e autônoma com uma linguagem própria favorável ao crescimento científico (Lopes, Szewczyk, Lunardi e Santos, 2007).

\section{SAE: Realidade ou Utopia na prática de en- fermagem?}

$O$ contato dos acadêmicos de enfermagem com a sistematização da assistência de enfermagem (SAE) é de vital importância para a formação teórico/prática deste alunado, pois traz para ele a familiaridade e a competência para a aplicação da sistematização, servindo como base do seu cuidado na vida profissional (Thofehrn, Traversi, Muniz, Duarte e Leite, 1999).

$A$ aplicabilidade da $S A E$ é realidade, entretanto, em pouquíssimos locais podemos visualizar a sua existência. Além disso, a mesma é feita de forma mecânica e repetitiva, sem ser dada a sua devida importância, estando até mesmo alguns impressos da SAE desatualizados (ACD. 4).

Eu vejo a aplicabilidade da SAE como realidade de um futuro não tão distante, no entanto é preciso que a classe de enfermagem lute com bastante ve- 
emência pela sua aplicabilidade, ou se não ela ficará apenas no papel e ai sim se tornará uma utopia (ACD.8).

A Enfermagem no Brasil vem transformando a concepção técnica da profissão em uma concepção relacional, ampliando e usando conhecimentos das mais diversas áreas para possibilitar a sistematização da assistência (Simões, 2007).

Ainda no que tange a perspectiva dos entrevistados notou-se que estes enxergam a SAE como uma utopia, por essa não se encontrar presente em sua totalidade nos campos de estágios e por não ser aderida completamente pelos profissionais que acompanham os acadêmicos.

Com base nas práticas vivenciadas durante a graduação, a SAE pode ser considerada uma Utopia, principalmente pela sobrecarga do profissional de enfermagem em nossas instituições de saúde haja vista a necessidade de desenvolver não só a assistência como ser responsável por diversas funções que afastam o enfermeiro do paciente (ACD.1).

Ainda é muito utópica a SAE aplicada por profissionais, se limitando a diagnósticos óbvios que não baseiam assistência nenhuma, servindo apenas para simular a $S A E$, visto que muitas vezes os profissionais de enfermagem acabam responsáveis por questões mais administrativas que de assistência (ACD.12).

A atuação do profissional de enfermagem, sobre tudo do enfermeiro, nem sempre condiz com as necessidades da pessoa assistida. Em muitas ocasiões essa atuação é direcionada para atividades que não são de competência da enfermagem, levando-a a execução de funções de outros profissionais e/ou de tarefas unicamente burocráticas que desviam a atenção da

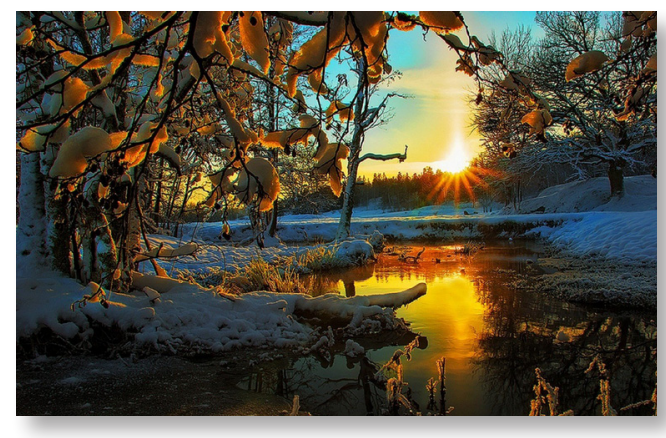

competência real do enfermeiro. Observamos que ao priorizar a função de outros profissionais em detrimento da sua própria função o enfermeiro subestima sua competência e a si mesmo como profissional (Faveri e Fernandes, 2003; Andrade e Vieira, 2005).

A dicotomia teórico/prática da SAE no processo de ensino aprendizado é amplamente evidenciada nos cursos de graduação, haja vista que o ensino da SAE, é algo recente e que na maioria das vezes é realizado isoladamente em cada disciplina, o que não facilita aos estudantes o aprendizado das habilidades necessárias para a sua aplicação.

Deve ser dito também que é de vital importância a articulação entre a academia e o serviço de saúde, entre o enfermeiro docente e o enfermeiro do assistente do serviço de saúde, para que possam juntos qualificar não só o cuidado prestado no ambiente de ensino, mas também qualificar a formação dos futuros profissionais que ali estão (Damaceno, Santos, Pivoto, Silva e Silveira, 2009).

\section{CONSIDERAÇÕES FINAIS}

Apesar de vivenciar as dificuldades da dicotomia teoria/prática que permeiam a $\mathrm{SAE}$, o alunado pesquisado expressa considerável compreensão sobre a importância da assistência sistematizada no avanço científico da profissão e na melhoria da qualidade dos serviços prestados. Contudo, é notório que alguns 
ainda estão imersos no modelo tecnicista da enfermagem se eximindo da responsabilidade profissional e social que sua formação exige.

Nesse caminhar acadêmico onde o método científico da profissão deve ser valorizado e praticado continuamente é possível constatar que a SAE encontra-se no desenrolar de sua complexidade, transitando entre a utopia e a realidade na academia e, sobretudo, nos serviços de saúde.

Acredita-se que essa abordagem no campo de ensino teórico e prático da SAE deve ser pensada como uma associação entre academia e o serviço de saúde de modo que as dificuldades encontradas em ambos sejam superadas.

\section{REFERÊNCIAS}

- Alfaro-Lefevre, R. (2014). Aplicação do processo de enfermagem: Fundamento para o raciocínio clínico. Porto Alegre: Artmed.

- Ângelo, M., Forcella, H.T., \& Fukuda, I.M.K. (1995). Do empirismo à ciência: A evolução do conhecimento de enfermagem. Rev. Esc. Enferm. USP, 29 (2), 211-23.

- Andrade, J.S., \& Vieira, M.J. (2005). Prática assistencial de enfermagem: problemas, perspectivas e necessidade de sistematização. Rev. bras. enferm, 58 (3), 261-265. Recuperado de: http://www.scielo.br/pdf/reben/v58n3/ a02v58n3.pdf.

- Cunha, S.M.B., \& Barros, A.L.B.L. (2005). Análise da implementação da sistematização da assistência de enfermagem, segundo o modelo conceitual de Horta. Rev. bras. enferm, 58 (5), 568-72. Recuperado de: http:// www.scielo.br/pdf/reben/v58n5/a13v58n5.pdf.

- Damaceno, R.C., Santos, S.S.C., Pivoto, F.L., Silva, B.T., \& Silveira, R.S. (2009). Sistematização da Assistência de Enfermagem: Importância atribuída por estudantes de enfermagem. Rev. enferm, UFPE on line, 3(3), 73-81. Recuperado de: http://www.ufpe.br/revistaenfermagem/ index.php/revista/article/view/158.

- Faveri, F., \& Fernandes, M.S. (2003). Função administrativa do enfermeiro: administração da assistência ou administração do serviço? Enferm. Atual, 3 (18), 32-36.

- Fialho, A.V.M., Pagliuca, L.M.F., \& Soares, E. (2002). Adequação da teoria de déficit de autocuidado no cuidado domiciliar à luz do modelo de Barnum. Rev. Latino-am. enferm, 10 (5), 715-720. Recuperado de: http://www.scielo.br/pdf/rlae/v10n5/v10n5a14.pdf.

- Garcia, T,R., \& Nobrega, M.M.L. (2009). Processo de enfermagem: da teoria à prática assistencial e de pesquisa. Esc. Anna Nery Rev. Enferm 13(1), 188 -193. Recuperado de: http://www.scielo.br/pdf/ean/v13n1/v13n1a26.pdf.

Horta, W.A. (1979). Processo de Enfermagem. São Paulo: EPU.

- Lopes, F.L., Szewczyk, M.S.C., Lunardi, V.L., \& Santos, S.S.C. (2007). SAE como um novo fazer na atividade cuidativa da enfermeira com base na complexidade de Edgar Morin. Cogitare enferm, 11(1),115-119. Recuperado de: http://ojs.c3sl.ufpr.br/ojs/index.php/ cogitare/article/view/8278/5787.

- Mcewen, M., \& Wills, E.M. (2015). Bases teóricas para enfermagem. São Paulo: Artmed.

- Minayo, M.C.S. (2014) O desafio do conhecimento: pesquisa qualitativa em saúde. São Paulo: Hucitec.

- North American Nursing Diagnosis Association. Diagnósticos de Enfermagem da NANDA: Definições $e$ Classificações 2015-2017. (2015). Porto Alegre: Artmed.

- Ojeda, B.S., Santos, B.R.L., \& Eidt, O.R. (2004). A integração ensino e assistência de enfermagem: delineando possibilidades para uma prática contextualizada. Acta paul. enferm, 17 (4), 432-438.

- Simões, S.M.F. (2007). O significado do pensar/fazer da prática do enfermeiro: uma revisão sistemática em artigos da REBEn 1932-1971. Esc. Anna Nery Rev. Enferm, 11 (3), 509-514. Recuperado de: http://www.scielo.br/pdf/ ean/v11n3/v11n3a17.pdf.

- Thofehrn, M.B., Traversi, M.S., Muniz, R.M., Duarte, A.C., \& Leite, M.P. (1999). O processo de Enfermagem no Cotidiano dos Acadêmicos de Enfermagem e Enfermeiros. Rev. gaúch. enferm, 20 (1), 69-79.

- Waldow, V. (1988). Processo de Enfermagem: teoria e prática. Rev. gaúch. enferm, 9 (1), 14-22. 